\title{
The Drag of Tapered Cantilever Airfoils
}

\author{
R. H. Upson and M. J. Thompson, Ann Arbor, Michigan
}

(Received August 6, 1934)

A relatively simple empirical equation is set up to fit the experimental values of profile drag determined from N.A.C.A. tests on uniform-section rectangular airfoils of varying thickness, camber, etc., and at varying lift coefficients. This is made the basis for determining the profile drag of the more general airfoil whose chord and section vary along its span, and specifically the practical case of straight taper in planform and thickness ratio. The recognized theoretical expression for induced drag is similarly put into more useable form, and combined with the profile drag to get the following complete expression for the drag coefficient of a tapered airfoil:

$$
\begin{aligned}
& C_{D}=[\mathrm{R} . \mathrm{N} .]_{m}{ }^{-0.15}\left(0.0065 \varphi+0.125 \psi t_{0}{ }^{2}\right)\left(1+0.7 C_{L^{3}}\right) \\
&+ 0.318 C_{L^{2}}\left(S / b^{2}+\gamma\right) \\
&
\end{aligned}
$$

where [R.N. $]_{m}$ is the Reynolds number based on the mean geometric chord; $t_{0}$ is the root thickness ratio; $b^{2} / S$ is the aspect ratio; and $\varphi, \psi$ and $\gamma$ are mathematical functions of the taper characteristics which have been put into convenient tabular and chart form. After showing that the equation checks closely with known experimental results for tapered airfoils, up to near the stall, numerical computation is extended to a series of cantilever airfoils, varying in taper characteristics, but related to each other on an assumed basis of structural equivalence. Interesting conclusions of practical importance are derived therefrom, showing particularly the value of tapering the planform almost to a point, the small importance of tapering the thickness ratio in such a case, and the comparatively large drag of the elliptical wing. It is incidentally brought out that the present criterion for the computation of wing bending moments is in error on the unsafe side for highly tapered wings; and an improved formula for lateral center of pressure is established, its distance from the wing root being given by: $\overline{\bar{x}}_{p}=b\left(0.185+0.085 K_{y}-0.020 K_{y}{ }^{2}\right)$ for straight tapered wings without twist; where $b$ is the span, and $K_{y}$ is the ratio of tip chord to root chord.
A $\mathrm{T}$ the present time there is a sufficient mass of wind tunnel data available on the drag of airfoils of rectangular planform so that it is possible to compute quite accurately the resistance of such wings at angles of attack below the burble point. ${ }^{1}$ In the case of tapered wings, however, an estimation of the drag is considerably more difficult. There is no satisfactory method for the determination of the profile drag which takes into account all of the factors involved, while the theoretical methods developed by Glauer $t^{2}$ for the calculation of the induced drag of tapered wings are quite unsuited to engineering use.

The present paper is concerned with the development of a method whereby the complete drag coefficient for a wing of any arbitrary taper in planform and in thickness, aspect ratio, and root thickness ratio is determined as a function of the Reynolds number based on the mean geometric chord. The results are applied to the calculation of several tapered wings for which

\footnotetext{
${ }^{1}$ G. J. Higgins, The Prediction of Airfoil Characteristics, N.A.C.A. T.R. No. 312 (1929).

${ }^{2}$ H. Glauert, Aerofoil and Airscrew Theory (1926), Chapter XI.
}

experimental data are available and excellent agreement is found between the wind tunnel tests and the computed values.

The method also lends itself readily to a consideration of the relative merits of the tapered wing as compared with other planforms from a combined aerodynamical and structural standpoint. Such an analysis is carried out and the superiority of highly tapered wings in this regard is definitely shown.

\section{Calculation of Profile Drag}

The basic assumption made in the present method for the calculation of the profile drag of a tapered wing is that the wing may be divided into a series of elementary chordwise strips each of which acts independently of the others. The drag of the entire wing may then be obtained by integration from test results on its component sections. Such a process is greatly facilitated by a simple empirical equation connecting the essential variables.

The first step is the determination of an expression for the profile drag coefficient of an elementary section of the wing. Making use of 
the large mass of data ${ }^{3}$ now available from the Variable Density Wind Tunnel of the National Advisory Committee for Aeronautics, at Reynolds numbers of approximately $3,000,000$, the following equation will be found to fit the family of symmetrical airfoils of varying thickness ratio $t$. At zero lift the minimum profile drag coefficient may be written in the form

$$
C_{D_{0} \text { min. }}=a_{1}+a_{2} t^{2} .
$$

With $a_{1}=0.0065, a_{2}=0.125$, this matches the observed points within the limits of experimental error, fits the condition $t=0$ better than the similar equation suggested in reference 3 , and by having one less term is more easily handled.

It should be mentioned here that the tests on which this formula is based are subject to several errors ${ }^{4}$ so that the values of $a_{1}$ and $a_{2}$ may be subject to future modification when more accurate test data are available. For this reason the analysis has been carried out using the algebraic coefficients in Eq. (1), and the numerical values are then introduced in the final results.

For a given value of $C_{L}$, Eq. (1) may be modified empirically so as to read

$$
C_{D_{0}}=\left(a_{1}+a_{2} t^{2}\right)\left(1+a_{3} C_{L^{3}}\right) .
$$

This expression represents approximately the envelope of the curves of $C_{D_{0}}$ against $C_{L}$ for airfoils of varying camber in the range below the stall. For the data given in reference 3, $a_{3}$ has the value 0.7 . The introduction of the term in $C_{L}{ }^{3}$ in Eq. (2) is not entirely rational since for negative lifts the drag would decrease instead of increase. However, the expression agrees very well with observed data and its use may therefore be justified on an empirical basis as long as we restrict ourselves to positive values of $C_{L}$.

In the case of a tapered wing, consideration must be given to the fact that the chord may decrease markedly from root to tip and that there may consequently be an appreciable change

\footnotetext{
${ }^{3}$ Jacobs, Ward and Pinkerton, The Characteristics of Seventy-Eight Related Airfoil Sections from Tests in the Variable Density Wind Tunnel, N.A.C.A. T.R. No. 460 (1933).

${ }^{4}$ Reference 3, pp. 45-46.
}

in the Reynolds number of the elementary wing strips of very highly tapered wings. It will now be assumed that the drag coefficient is related to the Reynolds number, R. N., by the exponential law

$$
C_{D_{0}}=h[\mathrm{R} . \mathrm{N} .]^{n} f\left(t, C_{L}\right) \text {. }
$$

This expression is not entirely satisfactory except for a flat plate set at zero angle of attack in which case the drag is pure skin friction. For the high Reynolds numbers considered in this work, the boundary layer is undoubtedly almost completely turbulent and $n$ has the value of $-1 / 5$ based on the assumption of a boundary layer velocity distribution which varies as the $1 / 7$ th power of the distance from the plate. Kármán, ${ }^{5}$ however, has pointed out that as the Reynolds number increases the exponent $1 / 7$ of the velocity distribution becomes smaller and smaller with a consequent change in the value of $n$. He has derived a logarithmic law for skin friction which is undoubtedly more exact than the exponential one but which is much too complicated to be used in this problem.

A more serious problem lies in the determination of the scale effect on the form drag for airfoils of finite thickness and camber. At present no satisfactory theory is available for the calculation of this effect and we are therefore again compelled to resort to empirical methods. Although such experimental data as exist are rather meager and incomplete, fairly satisfactory agreement with wind tunnel tests is obtained if Eq. (3) is used for the entire profile drag coefficient and $n$ is put equal to -0.15 .

The profile drag coefficient of any element of the wing may thus be written as

$$
C_{D_{0}}=h[\mathrm{R} . \mathrm{N} .]^{n}\left(a_{1}+a_{2} t^{2}\right)\left(1+a_{3} C_{L^{3}}\right),
$$

where R.N. $=V y / \nu=\rho V y / \mu, y$ being the chord of the section, $V$ the velocity of flight, $\nu$ the kinematic coefficient of viscosity, $\rho$ the mass density of the air, and $\mu$ its absolute viscosity coefficient. At the standard temperature of $60^{\circ} \mathrm{F}$, $\mu$ has the value $3.78 \times 10^{-7}$. The value of $h$ is determined by the fact that if [R.N. $]_{e}$ is the Reynolds number of the experiments on which

\footnotetext{
${ }^{5}$ Th. von Kármán, Turbulence and Skin Friction J. Aero. Sci. 1, 1 (1934).
} 


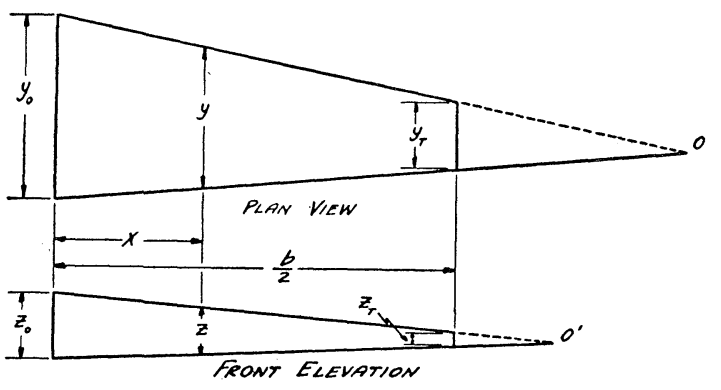

FIG. 1. Tapered wing diagram.

the values of $a_{1}, a_{2}$ and $a_{3}$ are based, then $h[\mathrm{R} . \mathrm{N} .]_{e}{ }^{n}=1$.

We are now prepared to take up the calculation of the profile drag of the tapered wing. Practical considerations usually demand a straight taper from the longitudinal axis out to near each tip. If $t$ varies from root to tip, the same requirement for straight elements usually applies, but this does not mean that $t$ itself varies uniformly. Referring to Fig. 1, let $a$ be the maximum thickness and $y$ the chord of any given section at a distance $x$ from the root. Then locally the thickness ratio is $t=z / y$. If the wing elements focus at 0 in plan view, then in general they will focus at $0^{\prime}$ in front elevation if $t$ is to be other than uniform. Neglecting any change of shape or rounding off at the tip, let

$K_{y}=y_{T} / y_{0} ; K_{z}=z_{T} / z_{0} ; K_{t}=t_{T} / t_{0}=K_{z} / K_{y}$,

where the subscripts $T$ and 0 designate the tip and root, respectively. If $b$ is the total span, then

$$
\begin{aligned}
& y=y_{0}\left[1-\frac{2 x\left(1-K_{y}\right)}{b}\right] \\
& z=z_{0}\left[1-\frac{2 x\left(1-K_{z}\right)}{b}\right] \\
& t=t_{0}\left[\frac{b-2 x\left(1-K_{z}\right)}{b-2 x\left(1-K_{y}\right)}\right]
\end{aligned}
$$

and

The drag coefficient for any section of the wing is obtained from Eq. (4), so that considering only the case of no aerodynamic twist, or in other words, a constant lift coefficient along the span, we get for the profile drag of the entire wing

$$
\begin{aligned}
D_{0} & =h\left(1+a_{3} C_{L^{3}}\right) q \int_{-b / 2}^{b / 2}\left(a_{1}+a_{2} t^{2}\right)\left(\frac{V y}{\nu}\right)^{n} y d x \\
& =2 h q y_{0}\left(\frac{V y_{0}}{\nu}\right)^{n}\left(1+a_{3} C_{L^{3}}\right) \int_{0}^{b / 2}\left[a_{1}+a_{2} t_{0}^{2}\left\{\frac{b-2 x\left(1-K_{z}\right)}{b-2 x\left(1-K_{y}\right)}\right\}^{2}\right]\left[1-\frac{2 x\left(1-K_{y}\right)}{b}\right]^{n+1} d x,
\end{aligned}
$$

where $q=\rho V^{2} / 2$ is the dynamic pressure. The profile drag coefficient for the entire wing is $C_{D_{0}}=D_{0} / q S$, where the wing area is $S=\left(y_{0} b / 2\right)$ $\times\left(1+K_{y}\right)$. We shall also find it most convenient to introduce the Reynolds number referred to the mean geometric chord, this latter quantity being $y_{m}=y_{0}\left(1+K_{y}\right) / 2$. After carrying out the integration we finally obtain

$$
C_{D_{0}}=[\text { R.N. }]_{m}{ }^{n}\left(\varphi a_{1}+\psi a_{2} t_{0}^{2}\right)\left(1+a_{3} C_{L}^{3}\right),
$$

where $[\text { R.N. }]_{m}=V y_{m} / \nu$,

$$
\begin{gathered}
\varphi=\frac{2^{n+1} h\left(1-K_{y}{ }^{n+2}\right)}{(n+2)\left(1+K_{y}\right)^{n+1}\left(1-K_{y}\right)}, \\
\psi=\frac{1}{\left(1+K_{y}\right)^{n+1}\left(1-K_{y}\right)}
\end{gathered}
$$

$$
\times\left[\psi_{1}-\left(1-\frac{1-K_{z}}{1-K_{y}}\right)\left(\psi_{2}-\psi_{3}\right)\right]
$$

and

$$
\begin{aligned}
& \psi_{1}=\frac{2^{n+1} h\left(1-K_{y}{ }^{n+2}\right)}{n+2}\left(\frac{1-K_{z}}{1-K_{y}}\right)^{2}, \\
& \psi_{2}=\frac{-2^{n+1} h\left(1-K_{y}{ }^{n}\right)}{n}\left(1-\frac{1-K_{z}}{1-K_{y}}\right), \\
& \psi_{3}=\frac{2^{n+2} h\left(1-K_{y}{ }^{n+1}\right)}{n+1}\left(\frac{1-K_{z}}{1-K_{y}}\right) .
\end{aligned}
$$

It will be noted at once that both functions $\varphi$ and $\psi$ become indeterminate as $K_{y}$ approaches unity, corresponding to the rectangular wing. The limiting value of $\sigma$ is easily found by 
differentiation to be $\operatorname{Lim} \varphi=h ; K_{y} \rightarrow 1$. In order to find the limiting value of $\psi$, we put $K_{y}=1-\lambda$ and expand in a power series in $\lambda$. We get after simplification

$$
\begin{aligned}
\psi= & \frac{2^{n+1} h}{(2-\lambda)^{n+1}}\left[\frac{\left(1-K_{z}\right)^{2}}{3}\left\{1-\frac{3 c_{1} \lambda}{4}+\frac{3 c_{2} \lambda^{2}}{10}-\frac{c_{3} \lambda^{3}}{12}+\frac{c_{4} \lambda^{4}}{56} \cdots\right\}\right. \\
& \left.-\left(1-K_{z}\right)\left\{1-\frac{2 c_{1} \lambda}{3}+\frac{c_{2} \lambda^{2}}{4}-\frac{c_{3} \lambda^{3}}{15}+\frac{c_{4} \lambda^{4}}{72} \cdots\right\}+\left\{1-\frac{c_{1} \lambda}{2}+\frac{c_{2} \lambda^{2}}{6}-\frac{c_{3} \lambda^{3}}{24}+\frac{c_{4} \lambda^{4}}{120} \cdots\right\}\right]
\end{aligned}
$$

where

$$
\begin{aligned}
& c_{1}=n-1, \\
& c_{2}=(n-1)(n-2), \\
& c_{3}=(n-1)(n-2)(n-3), \\
& c_{4}=(n-1)(n-2)(n-3)(n-4),
\end{aligned}
$$

so that

$$
\begin{aligned}
\operatorname{Lim}_{\lambda \rightarrow 0} \psi & =h\left[\left(1-K_{z}\right)^{2} / 3-\left(1-K_{z}\right)+1\right] \\
& =(h / 3)\left(1+K_{z}+K_{z}^{2}\right),
\end{aligned}
$$

which agrees with what would be obtained directly for the rectangular wing.

For the data considered in this paper we have, as mentioned on page 169, $a_{1}=0.0065, a_{2}=0.125$, $a_{3}=0.7$ when $R_{e}=3,000,000$, so that $h=9.366$ while we shall take $n=-0.15$. The corresponding values of $\varphi$ and $\psi$ for various values of $K_{y}$ and $K_{z}$ are given in Table I and are plotted in Fig. 2. In determining the values of $\psi$, it was found that in order to get satisfactory results when using the expression (7) it was necessary to carry through the computations with a large number of significant figures for values of $K_{y}$ near unity. For this reason the expansion in terms of $\lambda$ given in (8) was used for the range $0.7 \leqslant K_{y} \leqslant 1$ while

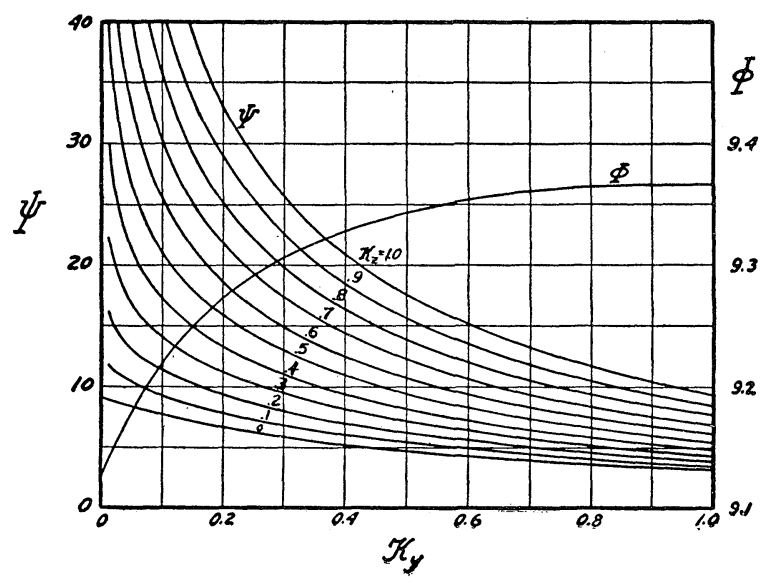

FIG. 2. Values of $\Psi$ and $\Phi$ for calculation of $C_{D_{0}}$. the exact formula ( 7$)$ was used for $0 \leqslant K_{y} \leqslant 0.7$. For $K_{y}=0.7$ the two formulas give results in entirely satisfactory agreement.

It will be noted that as $K_{y}$ approaches zero, the value of $\psi$ becomes infinite (except for $K_{z}=0$ ). These cases, of course, are of no practical significance since they correspond to wings having full taper in planform but only partial taper in thickness. The wing element at the tip thus becomes a section of infinite thickness ratio, and according to our original assumption its profile drag is infinite. Obviously this analysis does not apply to such extreme cases, since Eq. (1) is valid only for thickness ratios up to about 35 percent.

It should also be mentioned that this analysis gives the true minimum profile drag only for symmetrical sections, but it is a very close approximation for all good sections of small camber. In any case, it is a more fundamental representation of the effect of thickness variations than to deal with the actual minimum drag of cambered sections. The latter are more properly dealt with through the medium of lift coefficient as has been done here.

It is thus apparent that the mean effective thickness ratio of a straight-tapered wing is not a simple arithmetic mean, nor yet the value corresponding to the position of the so-called mean aerodynamic chord employed in center of pressure relations. The latter is the chord through the lateral centroid of area corresponding to

$$
\bar{x}=b\left(1+2 K_{y}\right) / 6\left(1+K_{y}\right),
$$

whereas the position of the section with mean effective thickness ratio could be obtained approximately (since $\varphi$ is practically constant) by putting $t=t_{0}(\psi)^{\frac{1}{2}}$ and solving for $x$, a totally useless procedure in this case. 
TABLE I. Values of $\varphi$ and $\psi$ for calculation of basic profile drag coefficient of tapered wings.

\begin{tabular}{|c|c|c|c|c|c|c|c|c|c|c|c|c|}
\hline \multirow[b]{2}{*}{$K_{y}$} & \multirow[t]{2}{*}{$\varphi$} & \multicolumn{11}{|c|}{$\psi$} \\
\hline & & $K_{2}=0$ & 0.1 & 0.2 & 0.3 & 0.4 & 0.5 & 0.6 & 0.7 & 0.8 & 0.9 & 1.0 \\
\hline 0 & 9.13 & 9.13 & $\infty$ & $\infty$ & $\infty$ & $\infty$ & $\infty$ & $\infty$ & $\infty$ & $\infty$ & $\infty$ & $\infty$ \\
\hline 0.01 & & $\begin{array}{l}8.94 \\
8.76\end{array}$ & $\begin{array}{l}11.70 \\
1120\end{array}$ & $\begin{array}{l}16.13 \\
14.70\end{array}$ & $\begin{array}{l}22.22 \\
1988\end{array}$ & $\begin{array}{r}29.99 \\
26.13\end{array}$ & $\begin{array}{r}39.43 \\
33.64\end{array}$ & $\begin{array}{l}50.55 \\
42.42\end{array}$ & $\begin{array}{l}63.33 \\
55.48\end{array}$ & $\begin{array}{l}77.77 \\
63.80\end{array}$ & $\begin{array}{l}93.89 \\
7.40\end{array}$ & $\begin{array}{r}111.67 \\
00026\end{array}$ \\
\hline $\begin{array}{l}0.02 \\
0.04\end{array}$ & & $\begin{array}{l}8.10 \\
8.45\end{array}$ & $\begin{array}{l}1.20 \\
10.53\end{array}$ & $\begin{array}{l}14.100 \\
13.52\end{array}$ & $\begin{array}{l}19.88 \\
17.43\end{array}$ & $\begin{array}{l}26.13 \\
22.25\end{array}$ & $\begin{array}{r}33.64 \\
27.98\end{array}$ & $\begin{array}{l}42.42 \\
34.63\end{array}$ & $\begin{array}{l}2.48 \\
42.20\end{array}$ & $\begin{array}{l}6.80 \\
50.68\end{array}$ & $\begin{array}{l}76.40 \\
60.06\end{array}$ & $\begin{array}{r}90.26 \\
75.09\end{array}$ \\
\hline 0.06 & & 8.16 & 10.02 & 12.61 & 15.94 & 20.01 & 24.81 & 30.34 & 36.61 & 43.61 & 51.35 & 59.83 \\
\hline 0.08 & & 7.90 & 9.59 & 11.91 & 14.84 & 18.40 & 22.58 & 27.38 & 32.80 & 38.84 & 45.50 & 52.78 \\
\hline 0.10 & 9.22 & 7.62 & 9.22 & 11.30 & 13.96 & 17.14 & 20.86 & 25.13 & 29.93 & 35.27 & 41.15 & 47.57 \\
\hline 0.20 & 9.27 & 6.64 & 7.79 & 9.27 & 11.08 & 13.22 & 15.68 & 18.47 & 21.59 & 25.04 & 28.81 & 32.91 \\
\hline 0.30 & 9.31 & 5.84 & 6.78 & 7.92 & 9.31 & 10.92 & 12.76 & 14.84 & 17.14 & 19.69 & 22.45 & 25.46 \\
\hline 0.40 & 9.33 & 5.24 & 6.00 & 6.93 & 8.04 & 9.33 & $\begin{array}{l}10.79 \\
\end{array}$ & $\begin{array}{l}1.04 \\
12.43\end{array}$ & $\begin{array}{l}1.14 .25 \\
\end{array}$ & $\begin{array}{l}16.09 \\
16.24\end{array}$ & 18.41 & 20.76 \\
\hline 0.50 & 9.34 & $\begin{array}{l}3.24 \\
4.73\end{array}$ & 5.37 & $\begin{array}{l}6.15 \\
6.15\end{array}$ & $\begin{array}{l}7.04 \\
7.08\end{array}$ & 8.14 & $\begin{array}{r}9.34 \\
\end{array}$ & $\begin{array}{l}10.69 \\
\end{array}$ & 12.17 & 13.80 & $\begin{array}{l}10.41 \\
15.57\end{array}$ & 17.47 \\
\hline 0.60 & 9.35 & 4.31 & 4.86 & 5.53 & 6.31 & $\begin{array}{l}0.14 \\
7.21\end{array}$ & 8.22 & 9.35 & 10.60 & 11.96 & 13.44 & 15.03 \\
\hline 0.70 & 9.36 & 3.94 & 4.51 & 5.00 & 5.68 & 6.45 & $\begin{array}{l}7.33 \\
\end{array}$ & 8.30 & 9.36 & 10.52 & 11.78 & $13: 13$ \\
\hline 0.80 & 9.36 & 3.63 & 4.06 & 4.57 & 5.16 & 5.84 & 6.59 & 7.44 & 8.36 & 9.36 & 10.45 & 11.62 \\
\hline 0.90 & 9.37 & 3.35 & 3.74 & 4.20 & 4.74 & 5.32 & 5.98 & 6.72 & 7.53 & 8.40 & 9.37 & 10.39 \\
\hline 1.00 & 9.37 & 3.12 & 3.47 & 3.87 & 4.34 & 4.87 & 5.46 & 6.12 & 6.84 & 7.62 & 8.46 & 9.37 \\
\hline
\end{tabular}

$K_{y}=$ tip chord/root chord; $K_{z}=$ tip thickness/root thickness.

\section{Calculation of the Induced Drag}

It is well known that the induced drag coefficient of any wing may be written in the form

$$
C_{D_{i}}=\left(C_{L}{ }^{2} S / \pi b^{2}\right)(1+\delta),
$$

where $\delta$ is dependent on the shape of the planform and is zero for an elliptical wing. The value of $\delta$ for the rectangular wing has been determined by Glauer $t^{6}$ as a function of the aspect ratio, while similar calculations have also been carried out for wings of any arbitrary taper in planform, but for on!y one aspect ratio, its value being equal to $\beta$, the lift curve slope for infinite aspect ratio. Now the angle of attack for the finite aspect ratio wing is, when measured in radians,

$$
\alpha=\alpha_{\infty}+\left(C_{L} S / \pi b^{2}\right)(1+\tau),
$$

where $\tau$, like $\delta$, is dependent on the planform shape. Differentiating with respect to $C_{L}$, we obtain for the lift curve slope

$$
\frac{d C_{L}}{d \alpha}=\frac{\beta}{1+(\beta / \pi)\left(S / b^{2}\right)(1+\tau)} .
$$

If the value of 0.072 is considered as an average value of $d C_{L} / d \alpha$ for rectangular wings of aspect ratio 6 , when the angle of attack is measured in degrees, then the above formula may be used to determine a suitable value for $\beta$. In this case we find, using Glauert's value for $\tau$, that $\beta=5.56$ ( $\alpha$ in radians), or 0.097 ( $\alpha$ in degrees).

The method employed by Glauert for the determination of the values of $\delta$ may, of course, be applied to tapered wings of any aspect ratio.

\footnotetext{
${ }^{6} \mathrm{H}$. Glauert, reference 2, ๆ 11.4-11.5.
}

The process as outlined in reference 2 finally reduces to the solution of a system of four linear equations in four unknowns, and in the general case of a wing of any taper and aspect ratio these equations would have algebraic coefficients. Even in particular cases where the coefficients may be reduced to numerical quantities, the process is a long and tedious one and for this reason we have adopted an approximate method of treating this phase of the problem.

In the case of the rectangular wing the value of $\delta$ considered as a function of aspect ratio may be represented approximately by the empirical equation

$$
\delta=0.049\left(b^{2} / S\right) / \beta=0.00881 b^{2} / S .
$$

The agreement with the computed values of $\delta$ could undoubtedly be improved by the use of a second degree equation but if it is remembered that $\delta$ always enters into the expression for the induced drag coefficient in the form $1+\delta$ and is usually small, it is seen at once that an error in the value of $\delta$ will produce only about onetenth of that error in $C_{D i}$. The above simplification thus appears to be entirely justified and Eq. (11) will be found satisfactory for aspect ratios from 4 to 16 .

Now for the tapered wing of aspect ratio equal to $\beta$, the value of $\delta$ as a function of $K_{y}$, the planform taper, is empirically

$$
\delta=0.141-0.404\left(K_{y}\right)^{\frac{1}{2}}+0.311 K_{y} .
$$

For tapered wings of any other aspect ratio, we shall assume that $\delta$ is affected by changes in aspect ratio alone in exactly the same way as 
the rectangular wing. Thus we may combine Eqs. (11) and (12) and write as an approximate general expression

$$
\delta=K\left(b^{2} / S\right)\left(0.141-0.404 K_{y^{\frac{1}{2}}}+0.311 K_{y}\right) .
$$

The constant $K$ is determined by the condition that when $b^{2} / S=\beta, K\left(b^{2} / S\right)=1$, so that we finally obtain

$$
\delta=\left(b^{2} / S\right)\left(0.0254-0.0727 K_{y^{\frac{1}{2}}}+0.0560 K_{y}\right) .
$$

The expression for the induced drag coefficient is now found to be

$$
C_{D_{i}}=\left(C_{L}^{2} / \pi\right)\left(S / b^{2}+\gamma\right),
$$

where $\gamma=0.0254-0.0727\left(K_{y}\right)^{\frac{1}{2}}+0.0560 K_{y}$. (See Table IV for numerical values.)

On inspection of Eq. (14), it is evident that a small part of the so-called induced drag coefficient has little or no dependence on aspect ratio but is exclusively a function of lift coefficient. The same applies to an important part of the socalled profile drag given by Eq. (7), except for the slight Reynolds number effect. The latter, as already pointed out, is of doubtful justification in the form used except for that portion of the drag directly due to skin friction. Hence there is no basic reason, beyond that of arbitrary definition, for classifying such parts of the drag as either profile or induced. But in view of the general acceptance of Glauert's values, approximately represented by Eq. (14), as "induced drag," we arbitrarily call the entire balance "profile drag" and choose our empirical equation to correspond. This was the classification assumed in working up all the results from reference 3 and should be kept in mind in the interpretation of other experimental data.

\section{Comparison with Experimental Results}

The complete drag coefficient for tapered airfoils may now be written as

$$
\begin{aligned}
C_{D}=[\text { R.N. }]_{m}{ }^{n}\left(\varphi a_{1}+\psi a_{2} t_{0}{ }^{2}\right) & \left(1+a_{3} C_{L}{ }^{3}\right) \\
+ & \left(C_{L}{ }^{2} / \pi\right)\left(S / b^{2}+\gamma\right),
\end{aligned}
$$

where $\varphi, \psi$ and $\gamma$ are obtained from Eqs. (7) and (14). Using the previously mentioned numerical values for the various constants (see page 171) we obtain

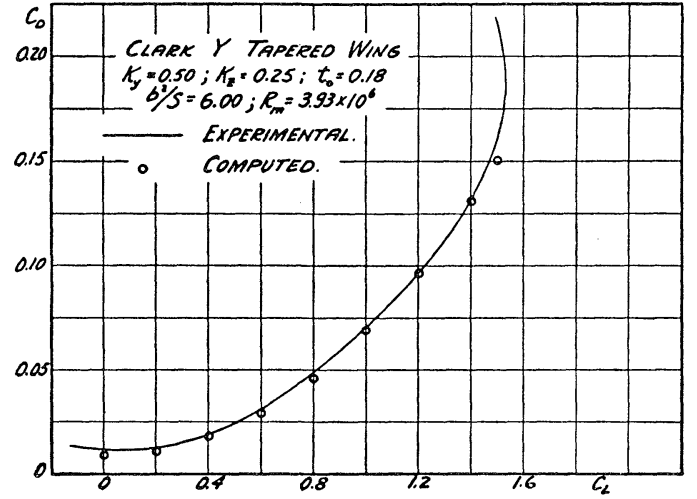

FIG. 3. Experimental and computed drag coefficients for tapered airfoils.

$$
\begin{aligned}
C_{D}=[\mathrm{R} . \mathrm{N} .]_{m}{ }^{-0.15}\left(0.0065 \varphi+0.125 \psi t_{0}^{2}\right) \\
\\
\times\left(1+0.7 C_{L}^{3}\right)+\left(C_{L}{ }^{2} / \pi\right)\left(S / b^{2}+\gamma\right) .
\end{aligned}
$$

In order to check the accuracy of the various assumptions on which our theory is based, computations have been carried out for several tapered wings for which experimental data exist. The results for the tapered Clark $Y$ wing $^{7}$ are shown in Fig. 3 and the agreement is found to be quite satisfactory except at and beyond the neighborhood of maximum lift. Comparisons with other tests in the variable density tunnel, including the case of a tapered wing with rectangular center section, ${ }^{8}$ show equally good agreement, but in the case of tests at low Reynolds numbers in atmospheric tunnels, such factors as turbulence would undoubtedly require a modification of the coefficients in the basic profile drag equation, and possibly a change in the exponent of the Reynolds number.

In connection with the expression for the complete drag coefficient given in Eq. (15), it should be noted that the quantities [R.N. $]_{m}^{-0.15}$ and $\varphi$ are almost constant for the usual conditions encountered in practice. Thus when the taper factors are once determined so as to give an optimum value of $\psi$, this equation is very appreciably simplified and readily lends itself to a further study of the effect of variations in design characteristics.

\footnotetext{
${ }^{7}$ R. F. Anderson, The Aerodynamic Characteristics of Three Tapered Airfoils Tesied in the Variable Density Wind Tunnel, N.A.C.A. T.N. No. 367 (1931).

${ }^{8}$ R. F. Anderson, Tests of Three Tapered Airfoils Based on the N.A.C.A. 2200, the N.A.C.A.-M6, and the Clark Y Sections, N.A.C.A. T.N. No. 487 (1934).
} 


\section{Relative Merit of Various Planforms}

From a purely aerodynamic point of view, the ideal wing would be one which for a given aspect ratio gave minimum values of $\varphi, \psi$ and $\gamma$. A practical comparison of different airfoils must, however, include consideration of certain structural factors. If we assume full depth wing beams in various cantilever wings, each carrying a concentrated load $W$ at mid-span to balance the distributed lift, the root bending moment must be

$$
M_{0}=(W / 2) \bar{x}_{p},
$$

where $\bar{x}_{p}$ is the lateral center of pressure distance from mid-span. The U. S. Department of Commerce requirements, in effect, make $\bar{x}_{p}$ equivalent to $\bar{x}$ in Eq. (9), subject to allowance for the width of the fuselage. Though this is a safe assumption for most wings hitherto in use, it is open to serious question for the highly tapered wings, as will now be apparent.

An exact expression for $M_{0}$ may be found for a tapered wing using the aerodynamic theory which forms the basis for induced drag calculations. ${ }^{9}$ According to Glauert's work given in reference 2 , the spanwise distribution of circulation along the wing may be represented approximately by the first four terms of a Fourier's series; that is,

$$
\begin{aligned}
\Gamma=2 b V\left(A_{1} \sin \theta+A_{3} \sin 3 \theta\right. & \\
& \left.+A_{5} \sin 5 \theta+A_{7} \sin 7 \theta\right),
\end{aligned}
$$

where $x=-(b / 2) \cos \theta$ and the coefficients $A_{1}$ $\cdots A_{7}$ depend on the shape of the planform and the aspect ratio. We now assume that the KuttaJoukovsky theorem holds for each elementary wing strip, so that $d L=\rho V \Gamma d x$ and the total lift is readily found to be

$$
L=\pi \rho V^{2} b^{2} A_{1} / 2 .
$$

In a similar way we may calculate the bending moment at the root section. We have

$$
\begin{aligned}
M_{0} & =\int_{0}^{b / 2} x d L \\
& =\left(\rho b^{3} V^{2} / 2\right)\left(A_{1} / 3+A_{3} / 5-A_{5} / 21+A_{7} / 45\right),
\end{aligned}
$$

\footnotetext{
${ }^{9}$ Similar calculations including both torsion and bending have been carried out with a somewhat different point of view by E. Amstutz, Calculation of Tapered Monoplane Wings, N.A.C.A. T.M. No. 578.
}

or introducing the lift from (17) and putting it equal to the total load $W$, we get

$$
M_{0}=\left(W b / \pi A_{1}\right)\left(A_{1} / 3+A_{3} / 5-A_{5} / 21+A_{7} / 45\right),
$$

so that

$$
\begin{aligned}
\bar{x}_{p}=(2 b / \pi)\left(\frac{1}{3}+A_{3} / 5 A_{1}-A_{5} / 21 A_{1}\right. \\
\left.+A_{7} / 45 A_{1}\right) .
\end{aligned}
$$

The values of the ratios $A_{3} / A_{1}, A_{5} / A_{1}$, etc., are readily calculated from the tables given in reference 2 . The values of $\bar{x}_{p} / b$ for rectangular wings of different aspect ratios are given in Table II.

TABLE II. Lateral center of pressure position vs. aspect ratio for rectangular wings.

\begin{tabular}{ccc}
\hline \hline$\left(b^{2} / S\right) / \beta$ & $\left(b^{2} / S\right)(\beta=5.56)$ & $\bar{x}_{p} / b$ \\
\hline 0.50 & 2.78 & 0.2225 \\
0.75 & 4.17 & 0.2250 \\
1.00 & 5.56 & 0.2275 \\
1.25 & 6.95 & 0.2290 \\
1.50 & 8.34 & 0.2310 \\
1.75 & 9.73 & 0.2320 \\
\hline \hline
\end{tabular}

It thus appears that $\bar{x}_{p}$ is practically independent of aspect ratio and in the remainder of this work, such an assumption will be made.

For the tapered wing of aspect ratio $b^{2} / S=\beta$ Glauert's values of $A_{1}, A_{3} \ldots$ etc., may be used to calculate $\bar{x}_{p} / b$ for different values of $K_{y}$. The results are given in Table III.

TABLE III. Lateral center of pressure position vs. planform taper for monoplane wing.

\begin{tabular}{lcc}
\hline \hline$K_{y}$ & $\bar{x}_{p} / b$ & $\bar{x} / b$ \\
\hline 0 & 0.1843 & 0.1667 \\
0.25 & 0.2045 & 0.2000 \\
0.50 & 0.2145 & 0.2222 \\
0.75 & 0.2220 & 0.2380 \\
1.00 & 0.2275 & 0.2500 \\
\hline
\end{tabular}

The values of $\bar{x} / b$ calculated from Eq. (9) are also given in Table III, and the results are plotted graphically in Fig. 4 as $2 \bar{x}_{p} / b$, the lateral center of pressure position.

From the obvious fact that the force distribution in the general case differs from the area distribution along the span, our original assumption of zero aerodynamic twist must now be called into question even for geometrically untwisted airfoils. To take the extreme case of 


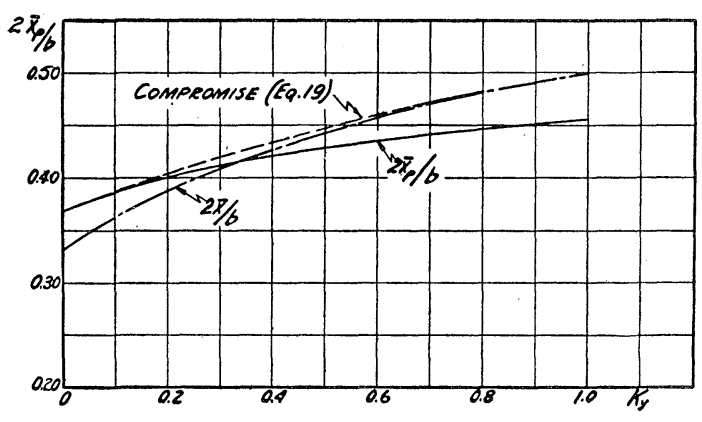

FIG. 4. Lateral center of pressure position $v$. planform taper.

taper to a point, the local lift coefficient at $b / 20$ from the tip is approximately 1.5 times the mean. The area to which this applies, however, is small; and at small values of $C_{L}$ the effect on the profile drag is small; while at large values of $C_{L}$ we must consider the three-dimensional flow and resultant burbling characteristics, which as yet are imperfectly known. Hence for profile drag purposes we shall continue to assume zero aerodynamic twist, which is equivalent to using an arithmetical mean of the lift coefficients. This can readily be shown to involve a negligible error in profile drag for a twist of any kind not exceeding about 5 degrees, except for the burbled condition above mentioned. The induced drag and lateral center of pressure for twisted airfoils can be taken from reference 2 .

Returning now to the airfoil without twist, i.e., chord lines all parallel, and median camber proportional to chord, it will be noted that the geometric and aerodynamic curves in Fig. 4 cross each other at $K_{y}=0.34$, approximately. For larger values of $K_{y}$, i.e., for planforms more nearly rectangular, the assumption of uniform pressure distribution, usually approximated at the burble point, is obviously the more severe case. The more pointed wings, however, show an intensification of pressure at the tips, which is more severe for the unburbled flow, and just opposite to the commonly assumed "tip loss." We shall therefore take a compromise curve of

$$
\bar{x}_{p}=b\left(0.185+0.085 K_{y}-0.020 K_{y}^{2}\right) .
$$

This single equation gives a good engineering approximation to the worst conditions of the other two curves, and is certainly simpler than Eq. (18).
Substituting (19) in (16) and dividing by $z_{0}$ we get the approximate root flange force $F$; then dividing by $W / 2$, and expressing the result in terms of aspect ratio and root thickness ratio $t_{0}$, we finally obtain a so-called structural quotient,

$$
\begin{aligned}
Q_{s}=\left(b^{2} / 2 S t_{0}\right)\left(1+K_{y}\right)(0.185+ & 0.085 K_{y} \\
& \left.-0.020 K_{y}^{2}\right) .
\end{aligned}
$$

For the rectangular wing, $K_{y}=1$ and this expression reduces to $Q_{s}=b^{2} / 4 S t_{0}$, while for the elliptical wing, the aerodynamic theory gives $A_{3}=A_{5}=A_{7}=0$, so that $\bar{x}_{p}=2 b / 3 \pi$, which is identical with the value for the lateral centroid of area. Thus the structural quotient for the elliptic wing becomes $Q_{s}=b^{2} / 6 S t_{0}$.

A given value of the structural quotient approximately establishes the root force in the spar flange as a proportion of the gross load carried, and seems a logical basis of comparison for cantilever wings, particularly when of constant span.

It will be of interest now to compare characteristics of a series of wings on the rather liberal structural basis of $Q_{s}=5$ and aspect ratio $b^{2} / S$ $=6$. Using the relations already laid down, we get the values shown in Table IV.

The wing proportions in the table have been arbitrarily chosen so as not to bring $t$ below 0.10 which is assumed as the structural minimum for torque stiffness. Below that point the saving in drag is small and is usually overbalanced by a more-than-proportional falling off of maximum lift.

It will be noted from Eq. (15) that an increase in $\gamma$ is exactly equivalent to a corresponding decrease in the reciprocal of the aspect ratio. Hence the latter can be used as a means of reducing the induced drag of all the airfoils to a common value. If the elliptical airfoil is arbitrarily chosen as the basis, each of the others can be brought into line by altering its $b^{2} / S$ factor, and finding the new value of $t_{0}$ which fits Eq. (20). $C^{\prime}{ }_{D_{0}}$ in the next to last column of Table IV is computed on this basis and thus forms a direct comparison of over-all merit from a drag standpoint. 


\section{Conclusions}

In spite of unconsidered factors, various conclusions as to tapered cantilever wings may be drawn from this table as follows :

A four to one planform taper with a uniform thickness ratio of 15.4 percent is structurally about as effective as an untapered wing of 30 percent thickness, and has about half the basic profile drag.

The further reduction in profile drag of a highly tapered wing, by superimposing a taper in thickness ratio, is comparatively slight. If, however, considerations of arrangement, vision or otherwise dictate a more moderate degree of planform taper, the benefits of taper in thickness ratio are more substantial (12 percent profile drag reduction for the wing with $K_{y}=0.5$ ), but the drag is greater than that obtained with more highly tapered planforms alone.

From a standpoint of performance alone, and without regard to construction economics, the elliptical planform is about equivalent to the twoto-one planform taper and decidedly inferior to a taper of four-to-one for the entire range of lift coefficient.

The taper to a point appears best of all in the final order of merit, though quantitatively the margin is small between this and the $8: 1$ taper. Also it is apparent that the larger span required to equalize the induced drag will increase the spar flange weight approximately in proportion to the span, for equal flange force If the thickness is further increased to maintain constant flange weight, the revised drag of the pointed airfoil is increased to approximately 0.0086 while the increase in the corresponding drag of the $8: 1$ airfoil is unnoticeable in the results as already given. On the other hand, minor considerations of distributed spar force and torsional stiffness theoretically favor the more pointed airfoil. Thus, though the absolute optimum for a specific case would call for a finite tip section, still it seems probable that tapers at least as high as $8: 1$ will be found advantageous for racing and large transports.

These conclusions on the relative merits of the highly tapered wing are, of course, based on the assumption that no difficulties will be encountered in obtaining satisfactory control and other necessary features. As the use of a highly 
tapered wing requires a large root chord, the problem of wing-fuselage interference may become acute, especially in the case of low wing monoplanes. Proper filleting at the wing root may solve this difficulty, but further experimental investigation is certainly required ${ }^{10}$. The same also applies to maximum lift.

${ }_{10}$ The case of a wing with $K_{y}=0.682$ has been studied by A. L. Klein, Effect of Fillets on Wing-Fuselage Interference, Trans. A.S.M.E. 56, No. 1 (1934).
Preliminary analysis of problems involving change in aspect ratio, load distribution, strut drag, etc., may be handled in a similar manner ${ }^{11}$ by the use of Eq. (15), assuming smoothly curved airfoil sections in the general range of proportions covered in reference 3 .

${ }^{11}$ For the effect of these and other variations on a complete airplane, see R. H. Upson, Wings-A Coordinated System of Basic Design, S.A.E. Journal, January, 1930; the coefficients being subject to further refinement as indicated in the present paper. 